\title{
Phase I and pharmacokinetic study of dasatinib and cetuximab in patients with advanced solid malignancies
}

\author{
Athanassios Argiris ${ }^{1}$, Trevor M. Feinstein ${ }^{1}$, Lin Wang ${ }^{2}$, Tianbing Yang ${ }^{3}$, Shruti Agrawal ${ }^{4}$, \\ Leonard J. Appleman ${ }^{1}$, Ronald G. Stoller ${ }^{1}$, Jennifer R. Grandis ${ }^{3}$, and Ann Marie Egloff ${ }^{3}$ \\ ${ }^{1}$ Division of Hematology-Oncology, Department of Medicine, University of Pittsburgh School of \\ Medicine, Pittsburgh, PA \\ 2Department of Pathology, University of Pittsburgh School of Medicine, Pittsburgh, PA \\ ${ }^{3}$ Department of Otolaryngology, University of Pittsburgh School of Medicine, Pittsburgh, PA \\ ${ }^{4}$ Bristol-Myers Squibb, New York City, NY
}

\begin{abstract}
Background-Combined inhibition of epidermal growth factor receptor (EGFR) and Src family kinases (SFK) may lead to improved therapeutic effects. We evaluated the combination of dasatinib, an inhibitor of SFK and other kinases, and cetuximab, an anti-EGFR monoclonal antibody.

Patients and Methods-Patients with advanced solid malignancies received cetuximab intravenously on a standard weekly schedule and dasatinib orally, once daily at 3 dose levels: (1) $100 \mathrm{mg}$, (2) $150 \mathrm{mg}$, (3) $200 \mathrm{mg}$. Pharmacokinetic and pharmacodynamic studies of dasatinib were performed prior to starting cetuximab and following 14 days of treatment.

Results-Twenty-five patients (3 dose level 1; 19 dose level 2; 3 dose level 3) were initially treated. Three patients developed dose-limiting toxicities: 1 at dose level 2 (headache) and 2 at dose level 3 (headache, nausea). Grade 3-4 toxicities in more than 2 patients included: dyspnea (4), vomiting (4), nausea (3), hypersensitivity reactions (3), headache (3) and anemia (3). Twentyone patients developed headache (8 grade $1 ; 10$ grade 2 ), which occurred after the loading of cetuximab and lasted 1-3 days. Six additional patients were treated with dasatinib starting 3 days after the loading dose of cetuximab; none developed headache after dasatinib. Dasatinib pharmacokinetics and a transient decrease in SFK PY416 levels in peripheral blood mononuclear cells were not altered by cetuximab. Patients with higher plasma TGF-alpha levels had worse progression-free survival.
\end{abstract}

Conclusions-Dasatinib $150 \mathrm{mg}$ once daily plus weekly cetuximab is recommended for phase II studies. Early-onset headache was ameliorated by starting dasatinib after cetuximab.

\section{Keywords}

dasatinib; cetuximab; Src; epidermal growth factor receptor; phase I; pharmacokinetic; pharmacodynamic

Corresponding Author: Athanassios Argiris, MD, Division of Hematology-Oncology, University of Pittsburgh, UPMC Cancer Pavilion, 5th Floor, Pittsburgh, PA 15232, Phone: 412-648 6575, Fax: 412-648-6579, argirisae@upmc.edu.

Conflict of interest statement

Drs. Argiris and Grand is have received research funding from BMS 


\section{Introduction}

Epidermal growth factor receptor (EGFR) has emerged as a promising anticancer therapy target for a variety of solid tumors. Cetuximab, a monoclonal antibody recognizing the EGFR ligand-binding domain, received approval by the United States (US) Food and Drug Administration (FDA) for the treatment of colorectal and head and neck cancers. Cetuximab has shown modest single-agent activity in head and neck and colorectal cancers and is well tolerated with rash, infusion reactions and hypomagnesemia as its most notable toxicities (1$3)$.

Preclinical data indicate that Src family kinases (SFK) mediate EGFR-dependent and independent signaling pathways important for tumor progression (4-7). Of the SFK, c-Src is most characterized and is overexpressed and/or activated in many epithelial cancers including lung and head and neck cancers (8). c-Src expression and activity increase at more advanced stages of tumor development and contribute to migration and invasion. Inhibition of SFK using pharmacologic inhibitors, siRNA or overexpression of a dominant-negative Src mutant was shown to inhibit EGFR transactivation and invasion (9). Importantly, combined SFK- and EGFR-targeted treatments was found to be more effective than either agent alone in reducing proliferation and invasion of SCCHN and survival of non-small cell lung cancer (NSCLC) in vitro (10-11).

Dasatinib, an inhibitor of several kinases, including SFK, BCR-Abl, c-Kit and EPHA2 (1214), is approved for the treatment of chronic myelogenous leukemia (CML) and Philadelphia chromosome-positive acute lymphoblastic leukemia. Recent data suggest that a once-daily schedule is equally efficacious to the twice-daily schedule initially used for CML and associated with lower incidence of pleural effusions (15). Preclinical data demonstrate that dasatinib inhibits cell migration and invasion in SCCHN and lung cancer cell lines (16). Moreover, preclinical models selected for resistance to cetuximab have been reported to be re-sensitized to cetuximab following dasatinib treatment (17), suggesting that the addition of a SFK-targeting agent to cetuximab therapy may improve clinical response.

\section{Patients and Methods}

\section{Patients}

Patients were enrolled in this study from June 2007 through April 2009. Main eligibility criteria included age $\geq 18$ years with advanced or refractory solid malignancies, an Eastern Cooperative Oncology Group (ECOG) performance status (PS) $\leq 2$, adequate organ function, QT interval corrected for heart rate (QTc) $\leq 450 \mathrm{msec}$ and no concomitant medications known to induce or inhibit CYP3A4. Previous radiotherapy or chemotherapy (not involving EGFR or SFK inhibitors) was permitted. The study protocol was approved by the University of Pittsburgh Institutional Review Board and registered with clinicaltrials.gov (NCT00388427). All participants provided written informed consent.

\section{Study treatment}

Cetuximab [supplied by Bristol-Myers Squibb (BMS), Princeton, USA] was administered weekly on days $1,8,15$ of a 21-day cycle. The first dose was $400 \mathrm{mg} / \mathrm{m}^{2}$ and all subsequent doses $250 \mathrm{mg} / \mathrm{m}^{2}$. Dasatinib (supplied by BMS) was administered orally at 3 dose levels: (1) $100 \mathrm{mg}$, (2) $150 \mathrm{mg}$, or (3) $200 \mathrm{mg}$ once daily on a continuous dosing schedule until disease progression. On cycle 1, dasatinib was started one day prior to cetuximab (day 0). Patients did not receive premedication with corticosteroids with the infusion of cetuximab. In an attempt to ameliorate early onset headache observed frequently in the first 25 enrolled patients, an additional 6 patients were enrolled and treated on an altered schedule: dasatinib treatment beginning 3 days after the first cetuximab dose of cycle 1 . 


\section{Evaluations}

Pretreatment assessment included history, physical examination, laboratory studies, electrocardiogram and tumor imaging studies within 4 weeks of registration. During the first cycle patients were evaluated weekly.

Toxicities were graded according to the National Cancer Institute Common Terminology Criteria for Adverse Events (version 3). Dose-limiting toxicity (DLT) was defined in the first cycle as grade 3 or higher non-hematological toxicity except the following grade 3 toxicities: nausea and vomiting, infusion reactions, rash and hypomagnesemia. Nausea or vomiting ( $\geq$ grade 3 ) lasting longer than 48 hours despite maximal medical therapy or a delay of more than 2 weeks in starting cycle 2 due to toxicity were considered DLTs. Patients with grade 3 or worse infusion reactions were removed from study. An abnormal non-hematological laboratory value (grade $\geq 3$ ) was considered a DLT if clinically significant and drug-related. DLT hematological toxicities were defined as absolute neutrophil count $<1000 / \mu \mathrm{L}$ lasting longer than 7 days, grade 4 thrombocytopenia or grade 3 or 4 neutropenia associated with sepsis or fever. Reassessment imaging, including chest imaging, was performed every 2 cycles ( 6 weeks) and response assessed by the Response Evaluation Criteria in Solid Tumors (RECIST) (18)

\section{Pharmacokinetic studies}

Dasatinib pharmacokinetic (PK) studies were performed on day 0 and on day 15 of cycle 1 for the standard treatment schedule cohort $(\mathrm{n}=25)$. Blood for PK analysis was collected at $0.5,1,2,3,4,5,8,10$ and 24 hours after dasatinib administration. On day 15 of the first cycle, cetuximab was administered 5-10 minutes following dasatinib dosing. Dasatinib in plasma was assayed at Bristol-Myers Squibb using liquid chromatography-coupled tandem mass spectrometry. Twenty-four samples were analyzed: 1 day 0 sample and 1 day 15 sample were inadequate for PK analysis. The following parameters were evaluated: maximum observed plasma concentration (Cmax), time of Cmax (Tmax), area under the plasma concentration-time curve from zero to the time of the last quantifiable concentration (AUC(0-T)) and terminal half-life (T-HALF), which was calculated as $\mathrm{Ln} 2 / \mathrm{Lz}$ (where $\mathrm{Lz}$ was the absolute value of the slope of the terminal log-linear phase). AUC(0-T) was calculated using the mixed log-linear trapezoidal algorithm in Kinetica. The lower limit of quantification for this assay was $1 \mathrm{ng} / \mathrm{ml}$.

\section{Pharmacodynamic studies}

P-SFK peripheral blood mononuclear cell (PBMC) levels-Activated SFK, SFK PY-416 (P-SFK), was measured in PBMCs just prior to dasatinib dosing and 2, 4, 8 and 24 hours after dasatinib dosing on days 0 and 15 of cycle 1 . Levels of P-SFK and c-Src were quantified in $50 \mu \mathrm{g}$ of PBMC protein lysate by quantitative immunoblotting with Odyssey Infrared Imaging System and software (LI-COR Biosciences) following incubation with anti-SFK PY-416 antibody (Cell Signaling) or anti-c-Src antibody (Upstate) diluted 1:500 and subsequent incubation with secondary antibody linked to RDye 680CW (1:5000, LICOR Biosciences).

Plasma analyte levels-Custom Searchlight ${ }^{\mathrm{TM}}$ (Thermo Scientific) multiplex enzymelinked immunosorbent assay (ELISA) was used to quantify EGFR, Transforming Growth Factor-alpha (TGF-a), amphiregulin (AR), hepatocyte growth factor (HGF), and cyclooxygenase-2 (COX-2) plasma levels in duplicate. A custom 2-plex assay for EGFR and HGF and custom 3-plex assay for TGF-a, AR and COX-2 were used according to the manufacturer's instructions. Independent reanalysis was performed for randomly selected $5 \%$ of samples in order to assess assay reliability. 
EGFR, P-SFK and c-Src levels in tumor tissues-Paired pre- and 3-5 week posttreatment tumor tissues were available for immunohistochemical (IHC) staining for 4 patients. Tissues were incubated with anti-EGFR (E3138, 1:75000; Sigma Diagnostics), anti-c-Src (SC-18, 1:100; Santa Cruz Biotechnology), or anti-P-SFK PY-416 (PK1109 1:50; Calbiochem) antibody with or without prior phosphastase treatment (P4978; Sigma) following antigen retrieval. EGFR staining was visualized using Mach 4 HRP-Polymer (M4U534H; Biocare Medical) and Dako Substrate Chromagen (K3468; Dako). P-SFK and c-Src were visualized using Dako Dual Envision (K4061; Dako) and Substrate Chromagen (K3468; Dako). Tumor staining intensity (integral scale from 0 to +3 ) and percentage (to the nearest $10 \%$ ) were interpreted by a pathologist.

\section{Statistical Methods}

The study followed a " $3+3$ " dose escalation design. Once the maximum tolerated dose (MTD) was determined, 12 additional patients were entered at the MTD to further study safety. Six additional patients were treated at the MTD with dasatinib starting 3 days after the loading dose of cetuximab.

The intraclass correlation coefficient (ICC) was used to assess ELISA reproducibility. Analyte correlations were evaluated using Pearson's correlation coefficient. Changes in analyte levels from baseline to 14 days after treatment were evaluated using the signed-rank test. Analyte level differences in patients who achieved stable disease (SD) versus those with progressive disease (PD) were evaluated using the rank sum test. High versus low levels of plasma analytes, defined by the median, were tested for association with progression-free survival (PFS), defined as time from first treatment on study to disease progression or death, using the log rank test. Plasma analytes whose levels were found to be significantly associated with PFS by the log rank test $(\mathrm{P}<0.05)$ were tested for association with PFS using univariate Cox proportional hazards models and multivariable Cox proportional hazards models adjusted for age and PS.

\section{Results}

A total of 31 patients were accrued (Table 1). Sixteen of the 25 patients treated on the original treatment schedule completed at least 1 cycle. Three patients did not complete the first cycle due to early disease progression and 6 patients because of toxicities, 5 of which occurred at the $150 \mathrm{mg}$ daily dasatinib dose and were not considered dose-limiting toxicities (DLT) according to predefined criteria. These non-dose-limiting toxicities included 3 hypersensitivity reactions (2 grade 3 and 1 grade 4 ), 1 grade 2 headache and 1 grade 3 nausea.. Patients received a median of 2 cycles (range: 1-15). Six patients had dasatinib dose reductions for treatment-related toxicities.

\section{Toxicities}

Among the 25 subjects treated on the original schedule, headache was the most common toxicity and was dose-limiting (Table 2). Typically, headache started the day of cetuximab loading and lasted 1-3 days (median 2 days). Headache was managed with analgesics, usually narcotics (e.g. oxycodone), and sumatriptan. Two patients required hospitalization for management of headache. Three patients developed DLT: 1 patient at a dasatinib dose of $150 \mathrm{mg} /$ day developed grade 3 headache; 2 patients at $200 \mathrm{mg} /$ day developed grade 3 headache and grade 3 nausea despite antiemetics. One patient developed a grade 4 anaphylatic reaction to dasatinib and another patient a grade 3 infusion reaction to cetuximab, which were not considered dose-limiting. Thus, the MTD was reached at 150 $\mathrm{mg} / \mathrm{day}$ of dasatinib. Pleural effusion developed after 2-5 months of treatment in 4 patients (grade 2 in 3 patients; grade 3 in 1 patient). Serious adverse events, grade 3 or 4 , reported in 
more than 2 patients included: dyspnea, vomiting, nausea, allergic reactions, headache and anemia (Table 3).

Of the 6 patients treated on the altered schedule, 3 experienced headache prior to starting dasatinib (grade 2 in 2 patients; grade 1 in 1 patient) which resolved before starting dasatinib on day 4. Other serious adverse events included hypophosphatemia $(n=2)$ and syncope with QTc prolongation $(n=1)$ (Table 3). One patient suffered sudden death at the doctor's office on cycle 1, day 21; no autopsy was performed, but the event was considered unlikely related to treatment.

\section{Pharmacokinetics}

The PK parameters (AUC and T-HALF) of dasatinib were comparable when given alone (day 0) or in combination with cetuximab (day 15) (Table 4). Dasatinib was rapidly absorbed with a Tmax of 1-2 hours. After the completion of the absorption phase, concentrations of dasatinib declined rapidly with a terminal phase half-life of 5-6 hours. Dasatinib Cmax and AUC parameters were similar for $100 \mathrm{mg}$ and $150 \mathrm{mg}$ dasatinib doses with and without cetuximab (Table 4).

\section{Antitumor activity}

In 23 patients evaluable for response, there were no objective responses; 10 patients with the following tumors had SD as best response: salivary gland cancer $(n=3)$, follicular thyroid carcinoma (1), papillary thyroid carcinoma (1), adenocarcinoma of the esophagus (1), sarcoma (2), adenocarcinoma of unknown primary (1) and NSCLC (1). Median duration of SD was 4.3 months (range 2-22). Patients with previously untreated adenocarcinoma of unknown primary and papillary thyroid carcinoma both had SD lasting 22 months. Data regarding tumor progression prior to study enrollment were not collected.

\section{Pharmacodynamics}

P-SFK and c-Src levels in PBMC protein lysates were measured (Figure 1A). Compared to baseline levels, P-SFK levels on day 0 tended to be reduced by 4 hours following dasatinib dosing, were significantly reduced by 8 hours and did not differ from baseline levels at 24 hours (Figure 1B). At day 15, P-SFK levels were also significantly decreased by 8 hours from same day pre-dasatinib dosing levels $(\mathrm{P}=0.03)$, and $\mathrm{P}-\mathrm{SFK}$ levels at 24 hours did not differ from pre-dasatinib dosing levels $(\mathrm{P}=0.18)$ (data not shown). Therefore, SFK were transiently inhibited in PBMCs following dasatinib dosing. The kinetics of inhibition were not appreciably altered with the co-administration of cetuximab. There was no association between antitumor activity and P-SFK levels.

P-SFK changes in tumor samples were heterogeneous. A NSCLC tumor had markedly reduced P-SFK levels post-treatment, while no appreciable changes in P-SFK levels was observed for the liposarcoma (Figure 1c) or parotid tumor (data not shown). The patient with liposarcoma had SD as best response while the other patients with paired tumor biopsies had PD as best response. c-Src was not altered with treatment (data not shown).

\section{Plasma analytes}

We measured plasma EGFR, TGF- $a$, AR, COX-2 and HGF levels in patients treated on the standard treatment schedule at baseline $(\mathrm{n}=25)$ and day $15(\mathrm{n}=16)$. COX-2 levels were generally below detection limit and were not further evaluated. ICCs for the remaining analytes were $93 \%$ to $98 \%$, indicating the assay performed well (data not shown).

AR and TGF- $a$ levels were significantly correlated at baseline and day $15(\rho=0.51, P=0.01$ and $\rho=0.60, P=0.02$, respectively). No other analytes tested were correlated. EGFR levels 
were reduced $(\mathrm{P}=0.007)$ and $\mathrm{AR}$ and TGF- $\mathrm{a}$ levels were increased $(\mathrm{P}=0.004$ and $\mathrm{P}=0.007$, respectively) after treatment; HGF levels were unaltered. Patients with PD had higher baseline TGF-a levels than patients with SD $(\mathrm{P}=0.02)$. Patients with high baseline TGF- $a$ levels had worse PFS $(\mathrm{P}=0.036)$ (Figure $2 \mathrm{~A})$. This association was statistically significantly in univariate proportional hazards models $(\mathrm{HR}=2.64 ; 95 \% \mathrm{CI}=1.03-6.78)$ but not after adjusting for patient age and performance status $(\mathrm{HR}=2.16$; 95\% $\mathrm{CI}=0.80-5.82)$. No other analyte tested was associated with disease progression (Figure 2).

\section{Discussion}

Approaches to enhance the antitumor activity of EGFR inhibitors are under active clinical investigation. The combination of EGFR and SFK inhibitors is well supported by preclinical data $(7,9-10)$, prompting our phase I trial. We demonstrated that $150 \mathrm{mg}$ daily dasatinib can be safely combined with cetuximab in patients with advanced solid malignancies and recommend $150 \mathrm{mg}$ daily dasatinib in combination with cetuximab for a phase II trial in SCCHN. Initiating dasatinib after cetuximab is recommended to avoid early-onset severe headache. The mechanism of headache remains unclear; a PK interaction was not demonstrated. PK studies showed no evidence that cetuximab affects dasatinib PK parameters, and the parameters observed were within the range reported for other dasatinib clinical trials. Dasatinib, a multi-kinase inhibitor, is currently under investigation in various solid malignancies. A recent phase II study of dasatinib monotherapy in NSCLC reported $43 \%$ of patients had either a partial response or SD (19). Low single agent activity was also reported in SCCHN (20), indicating it may be preferable to study dasatinib in combination regimens. The combination of erlotinib, an oral EGFR-tyrosine kinase inhibitor, and dasatinib has been investigated in a phase I/II trial in NSCLC with similarly common GI, fatigue and skin rash toxicities but with only one patient reporting headache, which was grade 2(21). Two partial responses were observed in the NSCLC trial combining erlotinib with dasatinub. One of these occurred in a patient whose tumor harbored an EGFR activating mutation. EGFR activating mutations, which render NSCLC sensitive to erlotinib, have not been reported US SCCHN patients.

P-SFK levels were decreased in PBMCs following dasatinib administration when dasatinib was given alone or after the addition of cetuximab. This transient decrease of P-SFK levels in PBMCs observed in patients is similar to the transient reduction in P-SFK PBMC levels seen in the nude mouse prostate xenograft model (22). We observed no correlation between degree of inhibition of SFK phosphorylation in PBMCs and disease control. Changes in PSFK levels in tumors with treatment were heterogenous. This may reflect transient inhibition of SFK as seen in PBMC and/or variable times from dasatinib dosing to tumor collection and fixation.

The changes in plasma EGFR, AR and TGF- $a$ levels following combined cetuximabdasatinib treatment likely reflect both physiological changes and the limitation of the ELISA assay. The accumulation of EGFR ligands following cetuximab administration in in vitro and in vivo cancer models was reported to result from ligand displacement and/or blocking of ligand binding sites by cetuximab(23). The observed decrease in EGFR plasma levels following treatment may reflect assay antibody specificity rather than physiological changes associated with treatment.

We are unable to distinguish the prognostic versus predictive value of baseline TGF-a plasma levels. However, baseline plasma TGF-a levels may be useful for defining patients more likely respond to this regimen. A planned phase II study in SCCHN patients combining dasatinib with cetuximab will assess the relationship between baseline TGF-a plasma levels and response. 


\section{Supplementary Material}

Refer to Web version on PubMed Central for supplementary material.

\section{Acknowledgments}

Funding: Supported in part by Bristol-Myers Squibb, and the Head and Neck SPORE Grant No. P50 CA097190-06 from the National Cancer Institute

Role of funding sources

BMS provided drug and support for the study costs and pharmacokinetics; the Head and Neck Cancer SPORE provided support for the conduct of the correlative studies.

\section{References}

1. Vermorken JB, Trigo J, Hitt R, Koralewski P, Diaz-Rubio E, Rolland F, et al. Open-label, uncontrolled, multicenter phase II study to evaluate the efficacy and toxicity of cetuximab as a single agent in patients with recurrent and/or metastatic squamous cell carcinoma of the head and neck who failed to respond to platinum-based therapy [see comment]. Journal of Clinical Oncology. 2007; 25(16):2171-7. [PubMed: 17538161]

2. Saltz LB, Meropol NJ, Loehrer PJ Sr, Needle MN, Kopit J, Mayer RJ. Phase II trial of cetuximab in patients with refractory colorectal cancer that expresses the epidermal growth factor receptor [see comment]. Journal of Clinical Oncology. 2004; 22(7):1201-8. [PubMed: 14993230]

3. Jonker DJ, O'Callaghan CJ, Karapetis CS, Zalcberg JR, Tu D, Au H-J, et al. Cetuximab for the treatment of colorectal cancer. The New England journal of medicine. 2007; 357(20):2040-8. [PubMed: 18003960]

4. Tice DA, Biscardi JS, Nickles AL, Parsons SJ. Mechanism of biological synergy between cellular Src and epidermal growth factor receptor. Proc Natl Acad Sci U S A. 1999; 96(4):1415-20. [PubMed: 9990038]

5. Maa MC, Leu TH, McCarley DJ, Schatzman RC, Parsons SJ. Potentiation of epidermal growth factor receptor-mediated oncogenesis by c-Src: implications for the etiology of multiple human cancers. Proc Natl Acad Sci U S A. 1995; 92(15):6981-5. [PubMed: 7542783]

6. Ishizawar R, Parsons SJ. c-Src and cooperating partners in human cancer. Cancer Cell. 2004; 6:209214. [PubMed: 15380511]

7. Egloff AM, Grandis JR. Improving Response Rates to EGFR-Targeted Therapies for Head and Neck Squamous Cell Carcinoma: Candidate Predictive Biomarkers and Combination Treatment with Src Inhibitors. Journal of oncology. 2009; 2009:896407. [PubMed: 19636423]

8. Summy JM, Gallick GE. Src family kinases in tumor progression and metastasis. Cancer Metastasis Rev. 2003; 22(4):337-58. [PubMed: 12884910]

9. Zhang Q, Thomas SM, Xi S, Smithgall TE, Siegfried JM, Kamens J, et al. SRC family kinases mediate epidermal growth factor receptor ligand cleavage, proliferation, and invasion of head and neck cancer cells. Cancer Res. 2004; 64(17):6166-73. [PubMed: 15342401]

10. Koppikar P, Choi SH, Egloff AM, Cai Q, Suzuki S, Freilino M, et al. Combined inhibition of c-Src and epidermal growth factor receptor abrogates growth and invasion of head and neck squamous cell carcinoma. Clinical Cancer Research. 2008; 14(13):4284-91. [PubMed: 18594011]

11. Leung EL-H, Tam IY-S, Tin VP-C, Chua DT-T, Sihoe AD-L, Cheng L-C, et al. SRC promotes survival and invasion of lung cancers with epidermal growth factor receptor abnormalities and is a potential candidate for molecular-targeted therapy. Molecular cancer research : MCR. 2009; 7(6): 923-32. [PubMed: 19491201]

12. Lombardo LJ, Lee FY, Chen P, Norris D, Barrish JC, Behnia K, et al. Discovery of N-(2-chloro-6methyl- phenyl)-2-(6-(4-(2-hydroxyethyl)- piperazin-1-yl)-2-methylpyrimidin-4-

ylamino)thiazole-5-carboxamide (BMS-354825), a dual Src/Abl kinase inhibitor with potent antitumor activity in preclinical assays. Journal of Medicinal Chemistry. 2004; 47(27):6658-61. [PubMed: 15615512] 
13. Wang XD, Reeves K, Luo FR, Xu LA, Lee F, Clark E, et al. Identification of candidate predictive and surrogate molecular markers for dasatinib in prostate cancer: rationale for patient selection and efficacy monitoring. Genome Biol. 2007; 8(11):R255. [PubMed: 18047674]

14. Li J, Rix U, Fang B, Bai Y, Edwards A, Colinge J, et al. A chemical and phosphoproteomic characterization of dasatinib action in lung cancer. Nat Chem Biol. 2010; 6(4):291-9. [PubMed: 20190765]

15. Kantarjian H, Cortes J, Kim DW, Dorlhiac-Llacer P, Pasquini R, DiPersio J, et al. Phase 3 study of dasatinib $140 \mathrm{mg}$ once daily versus $70 \mathrm{mg}$ twice daily in patients with chronic myeloid leukemia in accelerated phase resistant or intolerant to imatinib: 15-month median follow-up. Blood. 2009; 113(25):6322-9. [PubMed: 19369231]

16. Johnson FM, Saigal B, Talpaz M, Donato NJ. Dasatinib (BMS-354825) tyrosine kinase inhibitor suppresses invasion and induces cell cycle arrest and apoptosis of head and neck squamous cell carcinoma and non-small cell lung cancer cells. Clin Cancer Res. 2005; 11(19 Pt 1):6924-32. [PubMed: 16203784]

17. Wheeler DL, Iida M, Kruser TJ, Nechrebecki MM, Dunn EF, Armstrong EA, et al. Epidermal growth factor receptor cooperates with Src family kinases in acquired resistance to cetuximab. Cancer Biol Ther. 2009; 8(8)

18. Therasse P, Arbuck SG, Eisenhauer EA, Wanders J, Kaplan RS, Rubinstein L, et al. New guidelines to evaluate the response to treatment in solid tumors. European Organization for Research and Treatment of Cancer, National Cancer Institute of the United States, National Cancer Institute of Canada. J Natl Cancer Inst. 2000; 92(3):205-16. [PubMed: 10655437]

19. Johnson FM, Bekele BN, Feng L, Wistuba I, Tang XM, Tran HT, et al. Phase II study of dasatinib in patients with advanced non-small-cell lung cancer. J Clin Oncol. 2010; 28(30):4609-15. [PubMed: 20855820]

20. Brooks HD, Glisson B, Lu C, Sabichi A, Johnson F, Ginsberg L, et al. Phase II study of dasatinib in the treatment of head and neck squamous cell carcinoma (HNSCC). J Clin Oncol. 2009; 27(15s):abstr 6022.

21. Haura EB, Tanvetyanon T, Chiappori A, Williams C, Simon G, Antonia S, et al. Phase I/II study of the Src inhibitor dasatinib in combination with erlotinib in advanced non-small-cell lung cancer. $J$ Clin Oncol. 2010; 28(8):1387-94. [PubMed: 20142592]

22. Luo FR, Barrett Y, Ji P, Holly P, McCann E, Rhyne P, et al. Dasatinib (BMS-354825) pharmacokinetics correlate with pSRC pharmacodynamics in phase I studies of patients with cancer: Results from CA180003. Journal of Clinical Oncology, 2006 ASCO Annual Meeting Proceedings Part I. Jun 20.2006 24(18S):3046 2006. Supplement.

23. Mutsaers AJ, Francia G, Man S, Lee CR, Ebos JM, Wu Y, et al. Dose-dependent increases in circulating TGF-alpha and other EGFR ligands act as pharmacodynamic markers for optimal biological dosing of cetuximab and are tumor independent. Clin Cancer Res. 2009; 15(7):2397405. [PubMed: 19276250] 

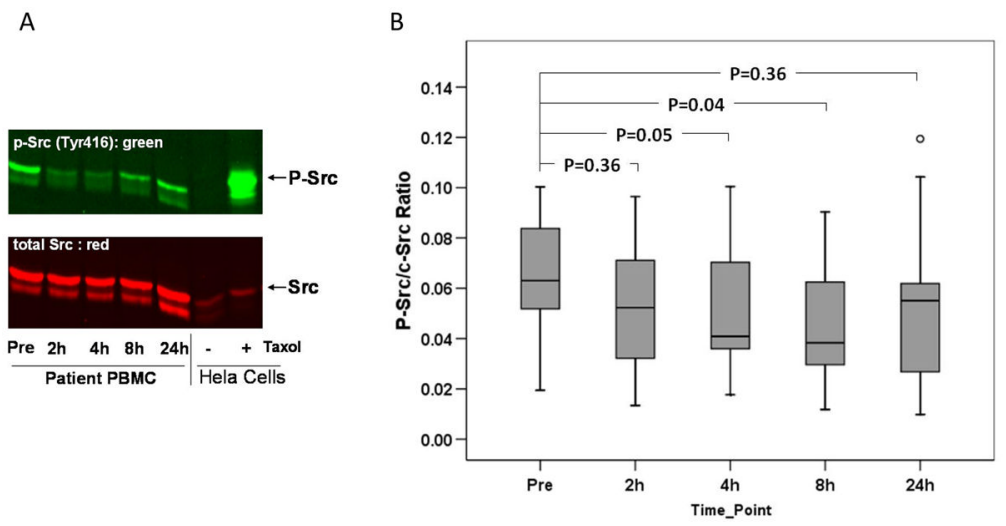

C

\section{Pre-Treatment}

\section{SFK PY-416}

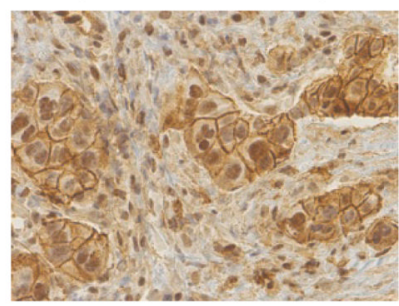

Patient 1-34 NSCLC

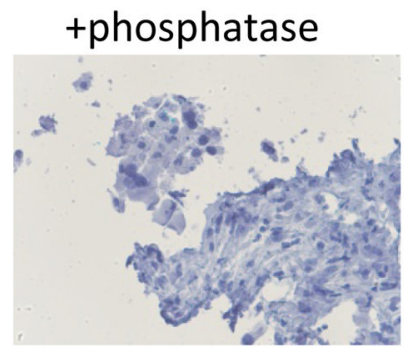

Post-Treatment

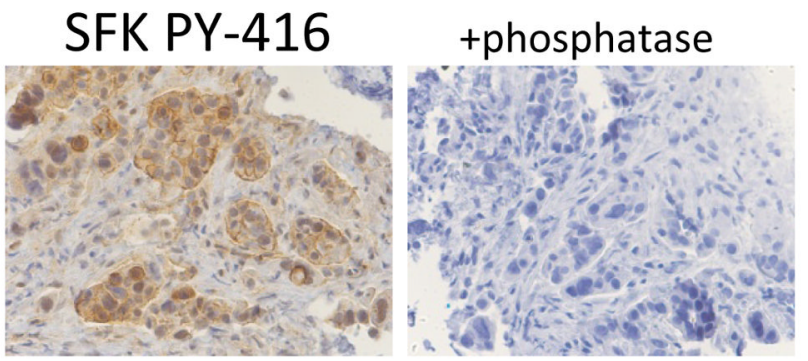

SFK PY-416

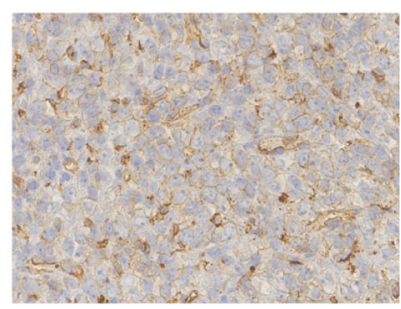

+phosphatase

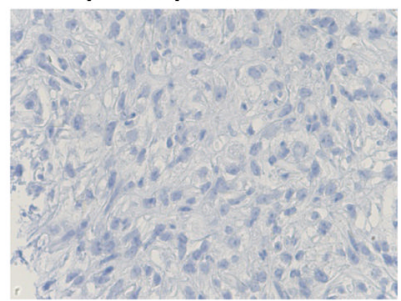

SFK PY-416

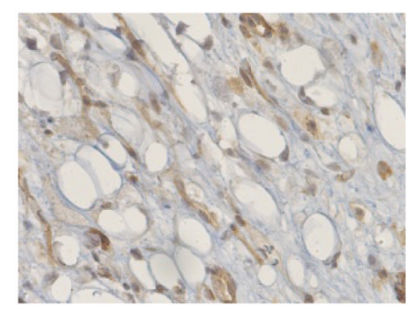

+phosphatase

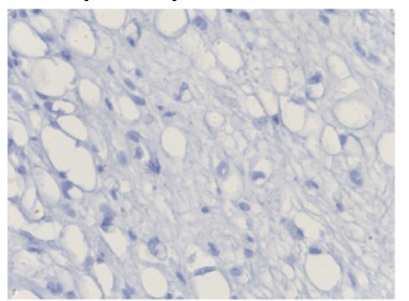

\section{Patient 1-40 Liposarcoma}

Figure 1. P-Src levels following dasatinib dosing were transiently decreased in PBMCs and varied in tumor tissues

(A) Image from Odyssey ${ }^{\mathrm{TM}}$ illustrating P-SFK and c-Src levels in PBMCs prior to dasatinib dosing (Pre) and at 2, 4, 8 and 24 hours after dosing. Hela cells with and without Taxol treatment were included as controls. (B) Distributions of P-SFK to c-Src ratios in patient PBMCs at day $0(n=21)$ prior to and at indicated hours following dasatinib dosing. Wilcoxon signed rank 2-tailed P values are presented. (C) P-SFK levels in paired pre- and post-treatment tissues. A NSCLC is presented in the top panels and liposarcoma in the bottom panels. Phosphatase treatment was included as a control. 
A

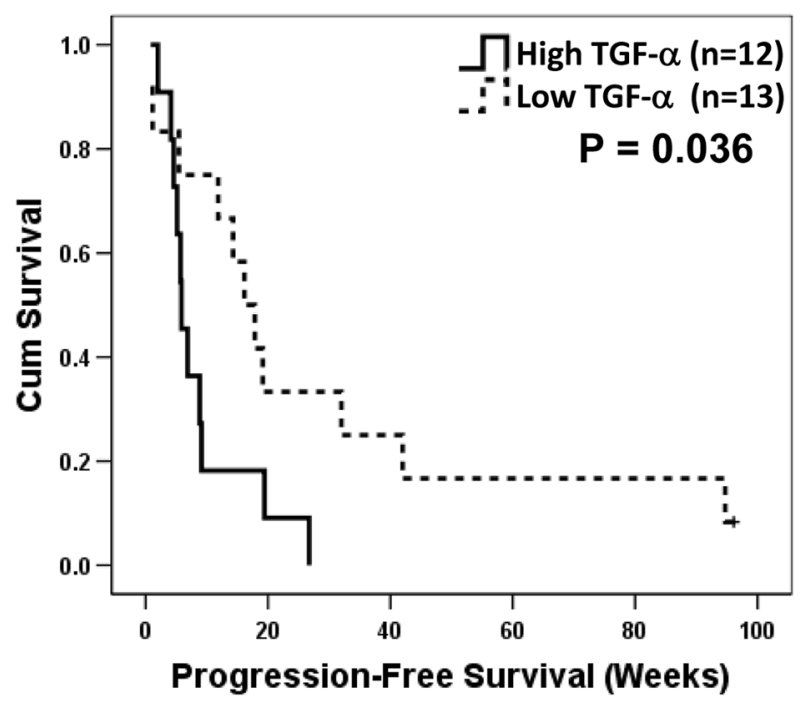

C

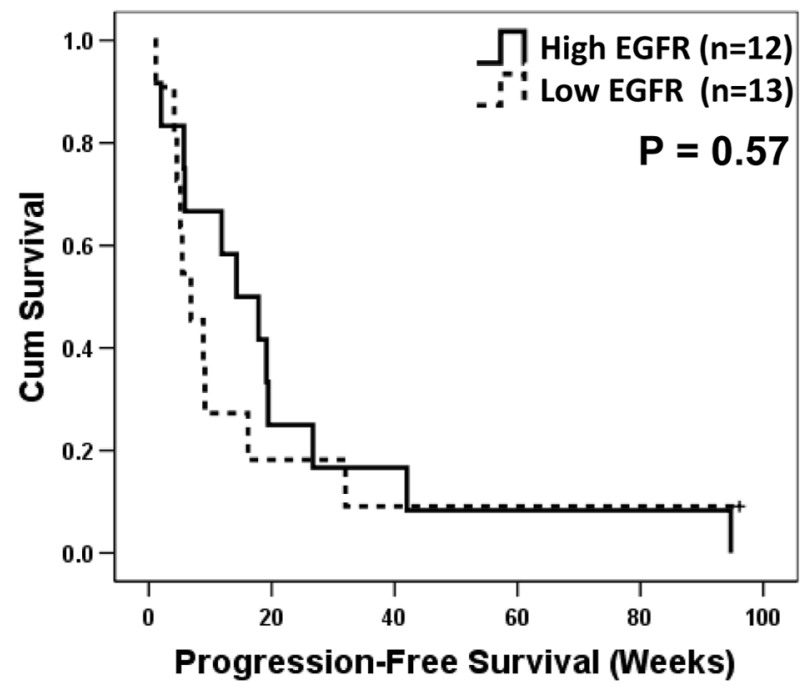

B

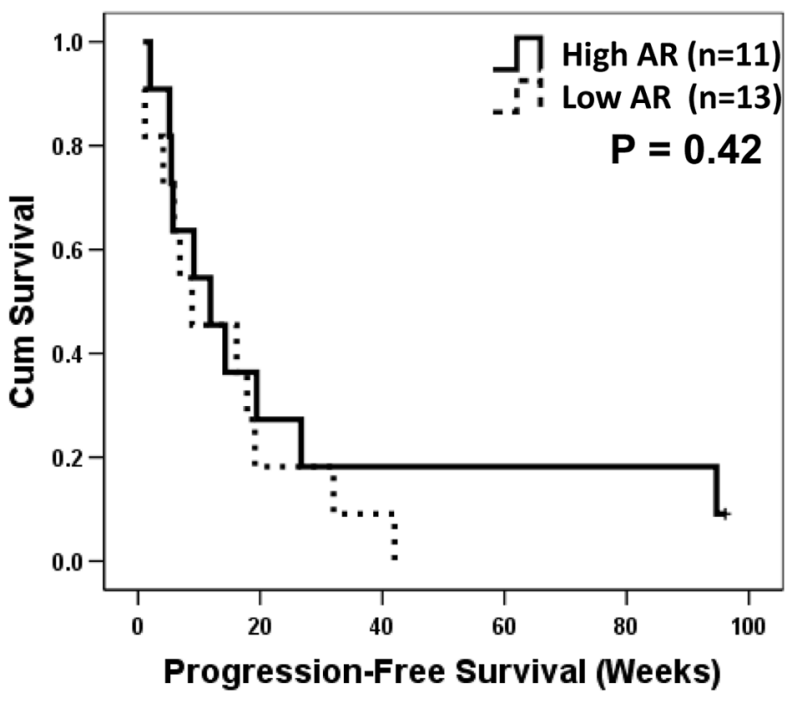

D

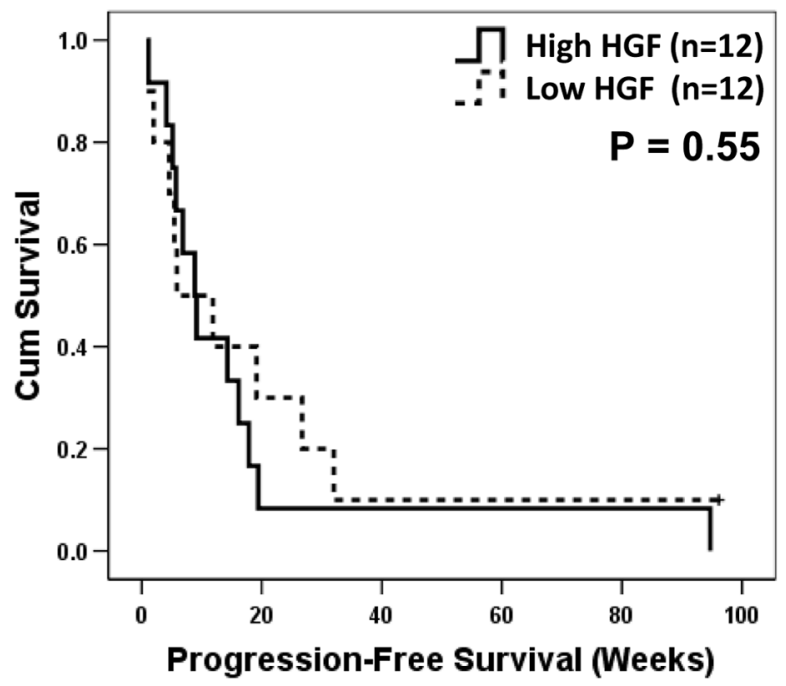

Figure 2. Lower plasma TGF-a levels were associated with improved progression-free survival Kaplan-Meier survival plots by baseline plasma levels of (A) TGF-a, (B) AR, (C) EGFR, and (D) HGF. High versus low levels were defined by the median level. Log rank P values are provided. 
Table 1

Patient characteristics

\begin{tabular}{|c|c|c|}
\hline & Standard schedule & Altered schedule \\
\hline No. of patients & 25 & 6 \\
\hline \multicolumn{3}{|l|}{ Gender } \\
\hline Male & 15 & 2 \\
\hline Female & 10 & 4 \\
\hline \multicolumn{3}{|l|}{ Age, years } \\
\hline Median (range) & $58(36-78)$ & $58.5(47-72)$ \\
\hline \multicolumn{3}{|l|}{ ECOG performance status } \\
\hline 0 & 15 & 0 \\
\hline 1 & 10 & 6 \\
\hline \multicolumn{3}{|c|}{ No. of previous chemotherapy regimens } \\
\hline Median (range) & $2(0-5)$ & $4(2-7)$ \\
\hline \multicolumn{3}{|c|}{ No. of previous biologic regimens } \\
\hline Median (range) & $0(0-3)$ & $0(0-0)$ \\
\hline \multicolumn{3}{|l|}{ Tumor types } \\
\hline Salivary gland & 6 & 0 \\
\hline NSCLC & 5 & 2 \\
\hline Small cell lung cancer & 1 & 0 \\
\hline Thyroid & 2 & 0 \\
\hline Colorectal & 2 & 0 \\
\hline Esophageal & 2 & 0 \\
\hline Other gastrointestinal & 3 & 0 \\
\hline Sarcoma & 2 & 2 \\
\hline Mesothelioma & 1 & 1 \\
\hline Bladder & 0 & 1 \\
\hline Unknown primary & 1 & 0 \\
\hline
\end{tabular}

Abbreviations: ECOG, Eastern Cooperative Oncology Group; NSCLC, non-small cell lung cancer 
Table 2

Patients with Dose-Limiting Toxicities (DLT)

\begin{tabular}{|cccc|}
\hline Dose Level & Dose of Dasatinib & Total No. of Patients (Evaluable) & DLT \\
1 & $100 \mathrm{mg}$ & $3(3)$ & None \\
2 & $150 \mathrm{mg}$ & $19(14)$ & 1 (Headache) \\
3 & $200 \mathrm{mg}$ & $3(2)$ & 2 (Nausea, Headache) \\
\hline
\end{tabular}




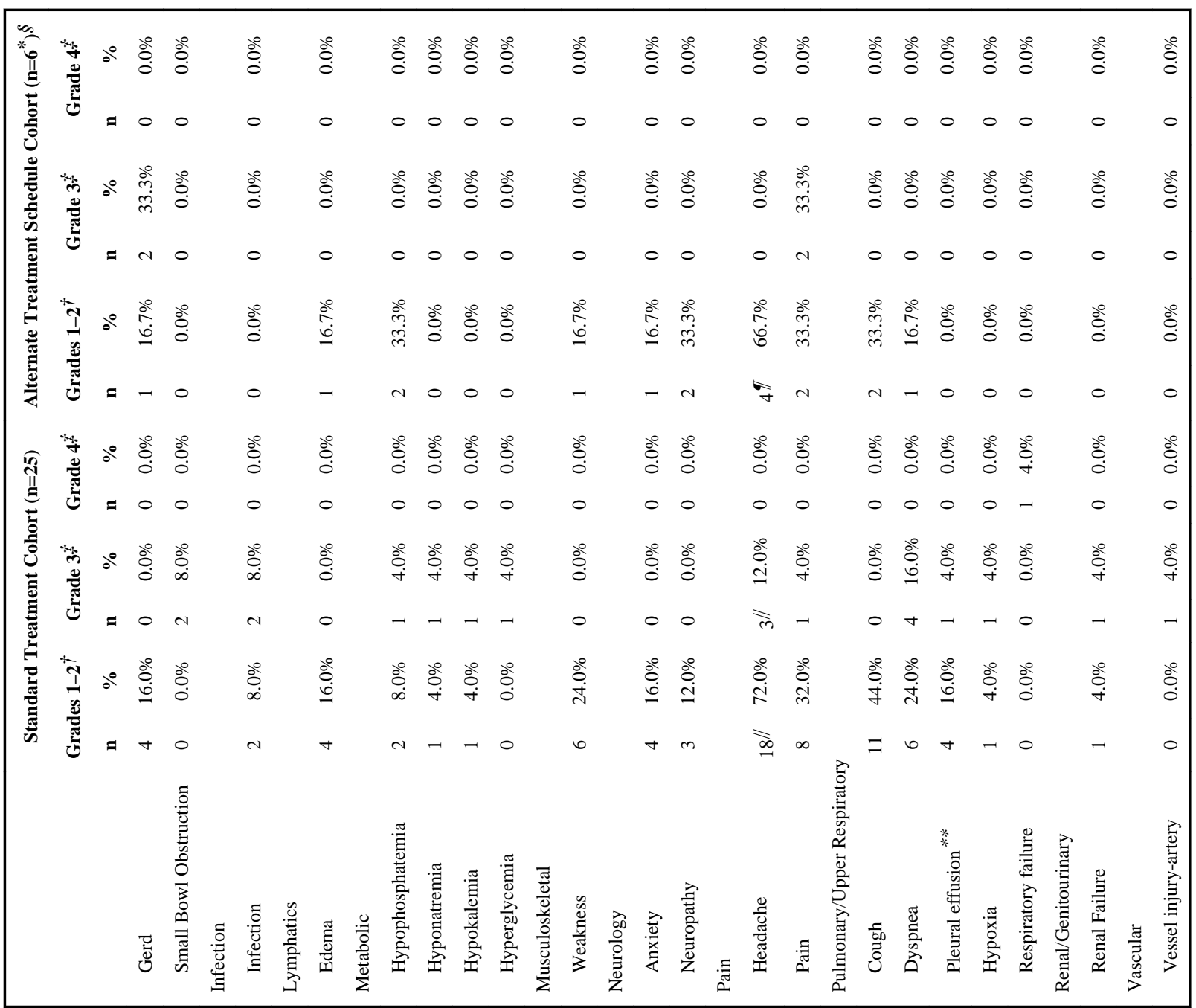




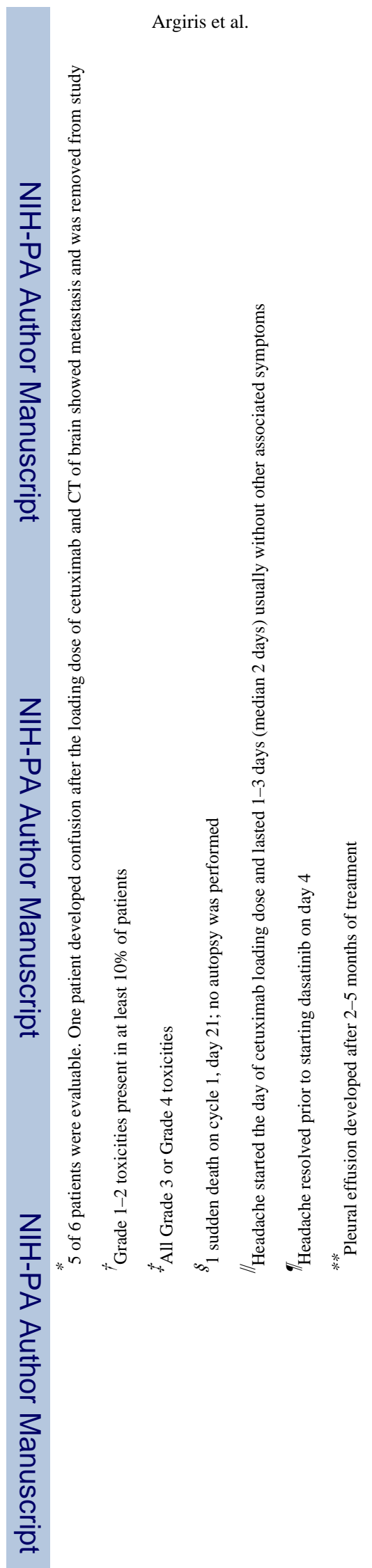

Invest New Drugs. Author manuscript; available in PMC 2013 August 01. 
